\title{
Changes in metabolites in the brain of patients with fibromyalgia after treatment with an NMDA receptor antagonist
}

The Neuroradiology Journal 0(0) 1-12

(C) The Author(s) 2019 Article reuse guidelines: sagepub.com/journals-permissions DOI: $10.1177 / 1971400919857544$ journals.sagepub.com/home/neu (SAGE

\author{
Nicolas Fayed ${ }^{1}$, Barbara Oliván ${ }^{2,8}$, Yolanda Lopez del Hoyo ${ }^{2,8}$, Eva Andrés ${ }^{3}$, \\ Mari Cruz Perez-Yus ${ }^{8}$, Alicia Fayed ${ }^{4}$, Luisa F Angel ${ }^{1}$, \\ Antoni Serrano-Blanco ${ }^{5,8}$, Miquel Roca ${ }^{6,8}$ and Javier Garcia Campayo ${ }^{7,8}$ (D)
}

\begin{abstract}
The aims of this work were to evaluate whether the treatment of patients with fibromyalgia with memantine is associated with significant changes in metabolite concentrations in the brain, and to explore any changes in clinical outcome measures. Magnetic resonance spectroscopy was performed of the right anterior and posterior insula, both hippocampi and the posterior cingulate cortex. Questionnaires on pain, anxiety, depression, global function, quality of life and cognitive impairment were used. Ten patients were studied at baseline and after three months of treatment with memantine. Significant increases were observed in the following areas: $\mathrm{N}$-acetylaspartate (4.47 at baseline vs. 4.71 at three months, $p=0.02)$ and $\mathrm{N}$-acetylaspartate+N-acetylaspartate glutamate in the left hippocampus (5.89 vs. 5.98; $p=0.007$ ); $\mathrm{N}$-acetylaspartate+ $\mathrm{N}$-acetylaspartate glutamate in the right hippocampus (5.31 vs $5.79 ; p=0.01)$ and the anterior insula (7.56 vs. 7.70 ; $p=0.033)$; glutamate+glutamine/creatine ratio in the anterior insula $(2.03$ vs. $2.17 ; p=0.022)$ and the posterior insula (1.77 vs. $2.00 ; p=0.004)$; choline/creatine ratio in the posterior cingulate $(0.18$ vs. $0.19 ; p=0.023)$; and creatine in the right hippocampus ( 3.60 vs. $3.85 ; p=0.007$ ). At the three-month follow-up, memantine improved cognitive function assessed by the Cognition Mini-Exam (31.50, $S D=2.95$ vs. 34.40, $S D=0.6 ; p=0.005)$, depression measured by the Hamilton Depression Scale $(7.70, S D=0.81$ vs. $7.56, S D=0.68 ; p=0.042)$ and severity of illness measured by the Clinical Global Impression severity scale $(5.79, S D=0.96$ vs. $5.31, S D=1.12 ; p=0.007)$. Depression, clinical global impression and cognitive function showed improvement with memantine. Magnetic resonance spectroscopy could be useful in monitoring response to the pharmacological treatment of fibromyalgia.
\end{abstract}

\section{Keywords}

Fibromyalgia, magnetic resonance spectroscopy, memantine

\section{Introduction}

Fibromyalgia (FM) is a chronic rheumatic condition of currently unknown aetiology that is characterised by the presence of diffuse musculoskeletal pain and painful sensitivity to touch in at least $11 / 18$ defined tender points. ${ }^{1,2}$ FM is one of the main health problems presently affecting Western countries because of its high prevalence, the clinical impact of patient disability and diminished quality of life and the significant health-care costs it produces ${ }^{3}$ It is currently accepted that FM treatments have limited efficacy, with an effect size of approximately 0.5 . $^{4}$

Pain is the most common and debilitating symptom of FM. Although the aetiological factors of the disease are not precisely known, the primary hypothesis on the pathogenesis of FM highlights the role of the central nervous system (CNS) in the amplification of pain perception (i.e. central sensitisation) and in the

${ }^{1}$ Department of Radiology, Quirónsalud Hospital, Spain

${ }^{2}$ Department of Psychology and Sociology, University of Zaragoza, Spain ${ }^{3}$ CIBER Epidemiology and Public Health, Clinical Epidemiology Unit, October 12 Hospital, Spain

${ }^{4}$ Department of Neurorehabilitation, San Juan de Dios Hospital, Spain ${ }^{5}$ Department of Psychiatry, Parc Sanitari St. Joan of God and the St. Joan of God Foundation, Spain

${ }^{6}$ Health Sciences Research University Institute, Juan March Hospital, Illes Balears University, Spain

${ }^{7}$ Department of Psychiatry, Miguel Servet Hospital and the University of Zaragoza. Aragon Institute for Health Research (IIS Aragon), Spain ${ }^{8}$ Preventative Activities and Health Promotion Network (REDIAPP) (G06/170)

\section{Corresponding author:}

Javier Garcia-Campayo, Miguel Servet University Hospital, Avda Isabel la Católica 1, 50009 Zaragoza, Spain.

Email: jgarcamp@gmail.com 
development of other co-morbid symptoms (originating in the CNS), such as sleep-related problems, fatigue, cognitive difficulties and emotional distress. ${ }^{5-7}$ Similarly, various studies have also reported reduced levels of certain neurotransmitters involved in the regulation of the descending analgesic response (i.e. serotonin and noradrenaline $)^{8}$ and increased levels of glutamate (Glu) and substance P. These abnormal levels of brain metabolites seem to be associated with increases in the pain response, facilitating hyperalgesia and allodynia, both characteristics of FM. ${ }^{9}$

In recent years, the neurophysiology of the pain process has led to increased interest in identifying the brain structures activated when patients experience pain through the use of different neuroimaging methods, such as positron emission tomography (PET), ${ }^{10}$ single photon emission computed tomography (SPECT), ${ }^{11,12}$ functional magnetic resonance imaging (fMRI) and, more recently, magnetic resonance diffusion tensor and volumetry. ${ }^{13}$

Proton magnetic resonance spectroscopy $\left({ }^{1} \mathrm{H}-\mathrm{MRS}\right)$ is one of the techniques used to assess potential disruptions of neuronal integrity and associated neurochemical dysregulations. MRS provides additional biochemical information which can be useful in determining the clinical stratification for a specific patient. ${ }^{14-18}$

NMDA receptor antagonists have neuroprotective properties that are important for pain reduction and are widely used in clinical practice. Dextromethorphan and ketamine have demonstrated efficacy in treating the pain of $F M,{ }^{19,20}$ although their long-term use shows significant limitations. Memantine, an NMDA receptor antagonist, is a non-competitive open-channel blocker that dissociates from the channel - a property that allows it to limit the pathological activity of the NMDA receptor without affecting normal synaptic activity. $^{21}$

Memantine has shown a very low incidence of side effects in human clinical trials, ${ }^{22,23}$ while a recent extension of trials has demonstrated the drug's clinical tolerability, even with prolonged use. ${ }^{24}$ The clinically approved dose of memantine for humans starts at $5 \mathrm{mg} /$ day, increasing progressively over the span of several weeks up to $20 \mathrm{mg} /$ day. This progressive dose adjustment may contribute to the drug's lack of side effects. ${ }^{22}$ Although this reduces NMDA receptor affinity, it contributes to the safety and efficacy of memantine as a neuroprotective agent. This quality, however, also makes memantine less effective for the treatment of chronic pain than high-affinity antagonists (e.g. ketamine). ${ }^{25}$ Nevertheless, recent research has highlighted the efficacy of memantine for the treatment of complex regional pain syndrome ${ }^{26}$ and phantom limb pain, ${ }^{27}$ which suggests that the quality of pain reduction depends on the type of pain being treated.

The aim of this study was to determine whether a three-month treatment of patients with FM with memantine is associated with significant changes in concentrations of metabolites - N-acetylaspartate
(NAA), N-acetylaspartate glutamate (NAAG) and creatine $(\mathrm{Cr})$ - and metabolite ratios - glutamate+glutamine/creatine $(\mathrm{Glx} / \mathrm{Cr})$ ratio and choline/creatine $(\mathrm{Cho} /$ $\mathrm{Cr}$ ) ratio - in the brain on MRS. The secondary aim was to explore whether changes in clinical outcome measures, such as cognitive performance, depression score, anxiety score and quality of life, are associated with the three-month treatment with memantine.

\section{Methods}

\section{Patients}

This was an open, uncontrolled, interventional, exploratory study with a three-month follow-up. Patients diagnosed with FM were recruited for inclusion in the study from primary health-care centres in Zaragoza, Spain. The patients were selected based on the following criteria: age 18-65 years, Spanish comprehension skills and diagnosed with FM by a rheumatologist certified by the American College of Rheumatology. Additional inclusion criteria $^{1}$ were having the patient sign an informed consent form and, for women, the use of birth control during the study.

The intervention consisted of administering $20 \mathrm{mg}$ memantine to 10 subjects. The treatment duration was three months, including four weeks of dose adjustment in which patients started with $5 \mathrm{mg}$ memantine in the first week and increased the dose by $5 \mathrm{mg}$ each week until the full dose was reached in the fourth week. The $20 \mathrm{mg}$ daily dosage continued for another two months. Medications permitted during the trial were those listed in the summary of product characteristics and the patient information leaflet for memantine. No other treatment for chronic pain was permitted. The study variables were evaluated at the start of the trial (baseline evaluation), at one month and at three months.

\section{Imaging methods}

MRI. The examinations were performed on a $1.5 \mathrm{~T}$ MRI HD clinical scanner (GE Healthcare Diagnostic Imaging, Milwaukee, WI). All of the images were acquired using an eight-channel phased-array head coil in both transmit and receive mode (NVHEAD A). All patients underwent brain T1 and T2-weighted MRI.

MRS studies. MRS studies were performed with the system automated single-voxel H1-MRS package (PROBE/SV; GE Medical Systems). Localised ${ }^{1} \mathrm{H}-\mathrm{MRS}$ was performed using a short echo time (TE) of $35 \mathrm{~ms}$, a repetition time (TR) of $2000 \mathrm{~ms}$ and 128 accumulations using a single voxel with a spin echo technique and selective excitation with gradient spoiling for water suppression.

Five $8 \mathrm{cc}(2 \mathrm{~cm} \times 2 \mathrm{~cm} \times 2 \mathrm{~cm})$ spectra were acquired on all patients, with automatic water suppression and placement of saturation bands to suppress lipid contamination. Spectra were rejected and repeated in the 
following cases: line width $>8 \mathrm{~Hz}$, line shape asymmetrical after eddy current correction and the presence of artifacts. The examinations were performed using a coronal T2-weighted image $(\mathrm{TR}=5350 \mathrm{~ms}$, $\mathrm{TE}=85 \mathrm{~ms}$, $90^{\circ}$ flip angle, number of excitations $=2$, matrix size $=320 \times 256$, field of view $=24 \mathrm{~cm} \times 24 \mathrm{~cm}$, slice thickness $/$ gap $=5 / 0 \mathrm{~mm}$ ) in the plane that goes through inner auditive conducts. The brain peduncle was used to locate volumes of interest (VOI; $2 \mathrm{~cm} \times$ $2 \mathrm{~cm} \times 2 \mathrm{~cm})$ in both hippocampi. A midsagittal T1-weighted image $\left(\mathrm{TR}=560 \mathrm{~ms}, \mathrm{TE}=12 \mathrm{~ms}, 90^{\circ} \mathrm{flip}\right.$ angle, number of excitations $=1$, matrix size $=256 \times$ 160 , field of view $=24 \mathrm{~cm} \times 24 \mathrm{~cm}$, slice thickness $/$ gap $=5 / 0 \mathrm{~mm}$ ) was obtained to locate a voxel in the posterior cingulate cortex (PCC). A line was drawn perpendicular to the splenium of the corpus callosum, and another line was drawn oblique to the surface of the corpus callosum. The intersection of the two lines was positioned above the lower corner of the voxel (Figure 1(a)). A parasagittal T1 image $(\mathrm{TR}=560 \mathrm{~ms}$, $\mathrm{TE}=12 \mathrm{~ms}, 90^{\circ}$ flip angle, number of excitations $=1$, matrix size $=256 \times 160$, field of view $=24 \mathrm{~cm} \times 24 \mathrm{~cm}$, slice thickness $/$ gap $=5 / 0 \mathrm{~mm}$ ) and $30 \mathrm{~mm}$ on the right, with respect to the plane of the corpus callosum. Since the insula has a triangular shape, we always try to place a voxel in the anterior and posterior insula. For the insula, the anterior edge was aligned with the anteroinferior insular pole in the axial plane; in the sagittal plane, the midline of the voxel was aligned with the
Sylvian fissure, the posterior edge of the voxel aiming at Heschel's gyrus. The medial edge of the voxel was aligned with the claustrum in the axial plane. The following exploration areas were chosen: (a) areas in which the authors have found increased Glu levels powered by $\mathrm{MRS},{ }^{28-30}$ (b) brain structures that are activated during painful conditions in healthy control group $^{31}$ and in FM patients ${ }^{32}$ and (c) regions (mentioned in prior reports) that have been implicated in cognitive impairment. ${ }^{33,34}$

The post processing of data was done with LCModel v6.2-0 (Stephen Provencher, Oakville, Canada), ${ }^{35}$ applying an eddy current correction and using an internal water signal reference to calculate the absolute metabolite concentrations. In addition to the individual analysis of the Glu, Cr, NAA and myoinositol ( $\mathrm{mI}$ ) compounds, we also studied the summed concentrations of the following three compound pairs: NAA+NAAG, referred to as total NAA; glycerophosphocholine+phosphocreatine (GPC), referred to as total Cho; and glutamate+glutamine, referred to as Glx (Figure 1(b)). The absolute metabolite values were only considered when the Kramer-Rao lower bound was $<20 \%$, thus indicating that these metabolites could be reliably estimated.

Concentration values are not corrected for cerebral spinal fluid contributions. We also obtained the absolute concentrations of brain metabolites and the ratios of the metabolites relative to $\mathrm{Cr}$. In clinical practice,

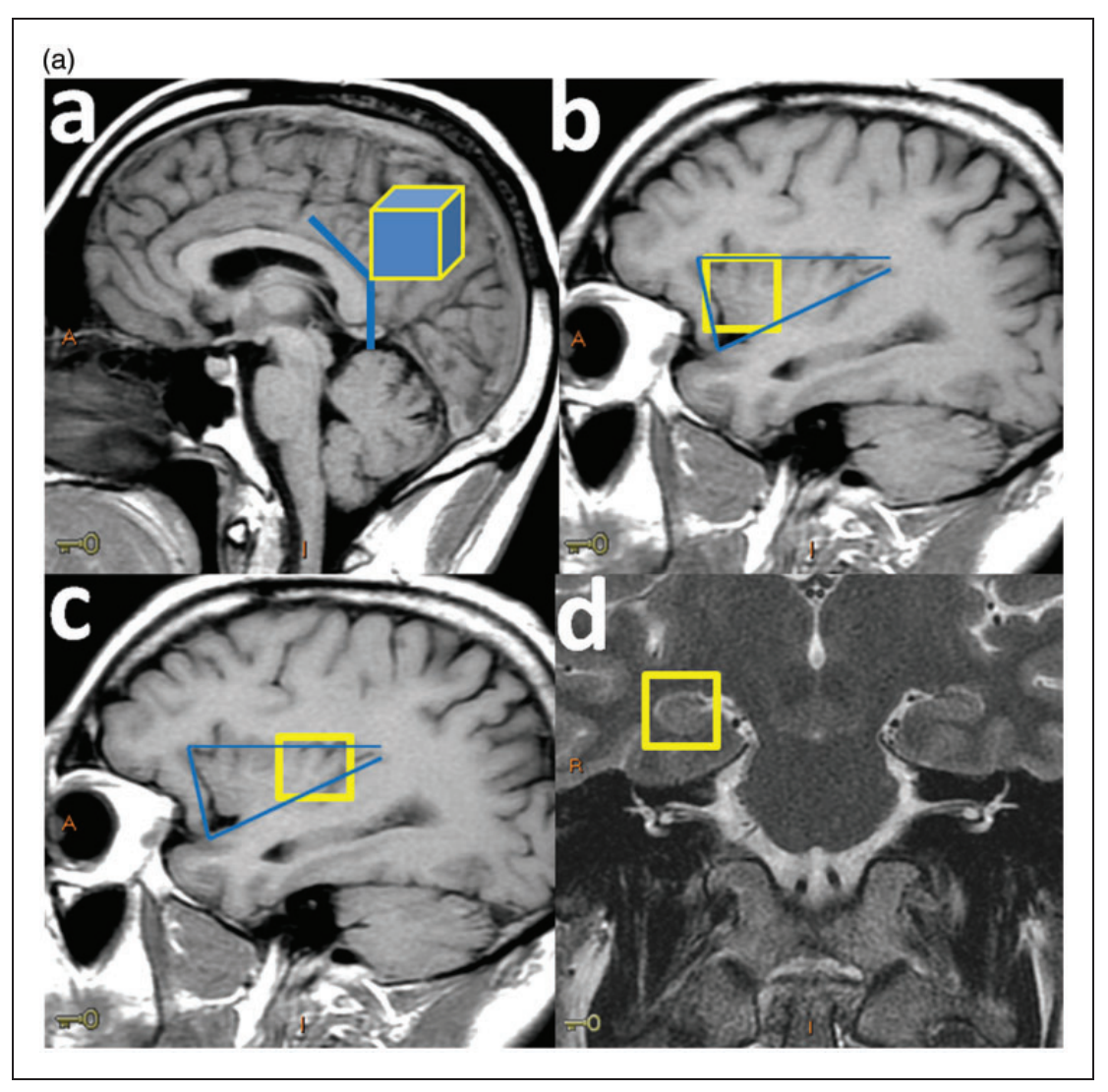

Figure 1(a). Voxel placement. Sagittal and coronal T1-weighted magnetic resonance imaging with the voxel placed in the (a) posterior cingulate gyrus; (b) anterior insula; (c) posterior insula; and (d) both hippocampi. 


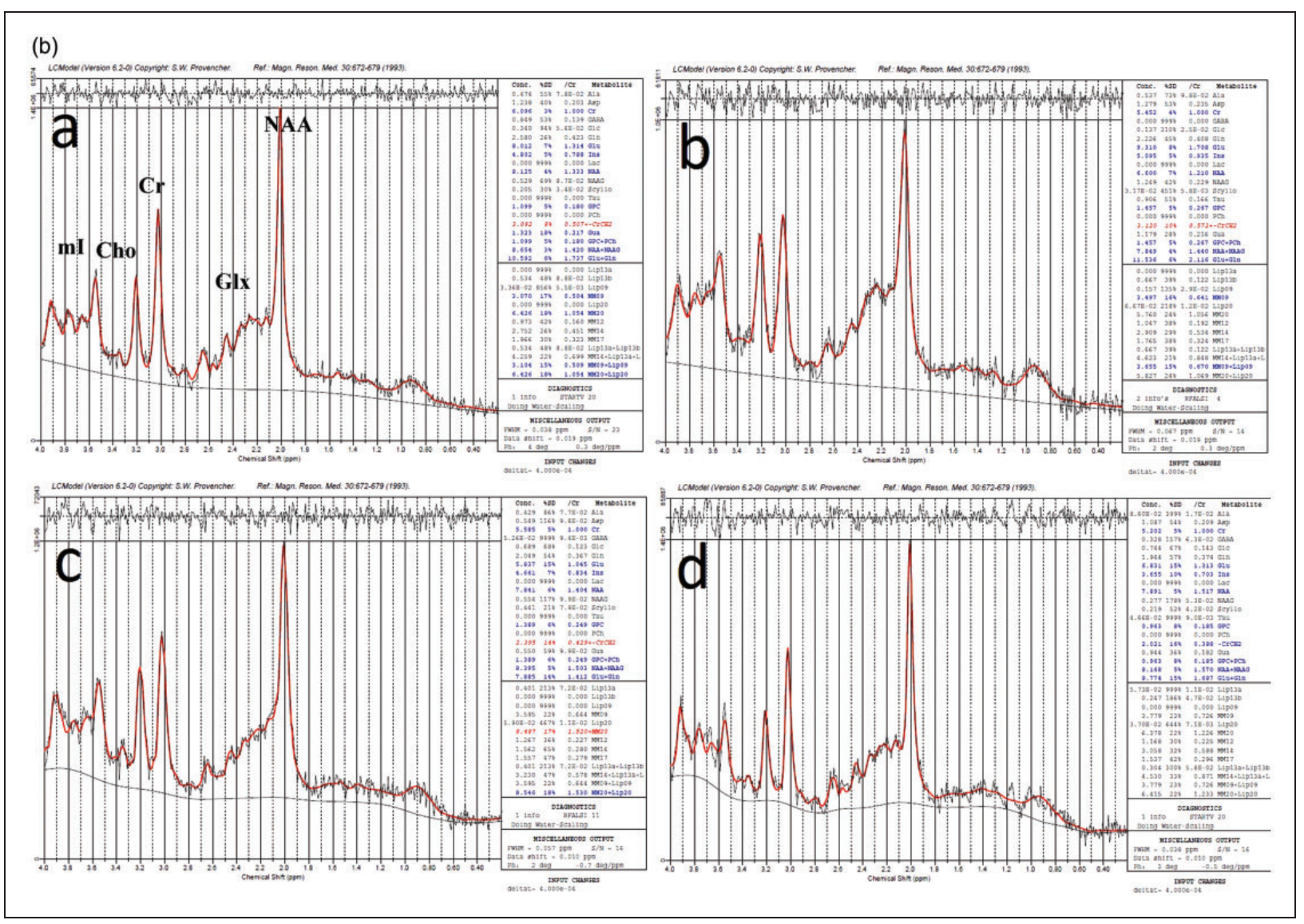

Figure 1(b). Example of spectrum acquired with the LCModel software in the (a) posterior cingulate gyrus; (b) anterior insula; (c) posterior insula; and (d) both hippocampi with the following peaks: ml: myo-inositol; Cho: choline compounds; Cr: creatine; Glx: glutamate+glutamine+GABA; NAA: N-acetylaspartate.

metabolic ratios are assessed using $\mathrm{Cr}$, which is considered the most stable metabolite, as an internal reference. $^{36}$

Secondary efficacy variables. The secondary efficacy variables were modifications to the following clinical variable values: pain threshold, pain perception, cognitive state, health status, anxiety and depression levels and quality of life. The variables were measured and assessed according to the following tests. Pain threshold was measured by a sphygmomanometer. ${ }^{37}$ Perceived pain was measured using a visual analogue scale (VAS). ${ }^{38}$ Cognitive state was measured using the Cognition Mini-Exam (MEC). In non-geriatric populations ( $<65$ years of age), such as the sample in this study, $\leq 27$ points is the threshold that suggests a 'likely case' of a cognitive disorder. The MEC test is the validated Spanish-language version of the Mini-Mental State Examination. ${ }^{39}$ FM health status was measured with the Fibromyalgia Impact Questionnaire (FIQ). ${ }^{40}$ The validated Spanish-language version of this questionnaire was used. ${ }^{41}$ Anxiety and depression levels were measured with the Hospital Anxiety Depression Scale (HADS). ${ }^{42}$ Quality of life was measured with the EuroQol 5D questionnaire. ${ }^{43}$ Severity of illness was measured with the Clinical Global Impression (CGI) scale, which is amongst the most widely used extant brief assessment tools in psychiatry. The CGI is a three-item observer-rated scale that measures severity of illness, global improvement or change and therapeutic response. $^{44}$

A small patient sample $(N=10)$ was chosen, as this was an exploratory study. Similar sample sizes have been used to identify significant differences in the levels of metabolites in different brain regions of FM patients and control groups. ${ }^{28-30}$

\section{Statistics}

To describe the quantitative variables, mean and standard deviations were calculated when they fulfilled normality criteria. The chi-square test was used for sociodemographic and other qualitative variables. The difference between clinical variables at baseline, one month and three months was calculated using an analysis of variance for repeated measures. The difference between neuroimaging tests taken at baseline and at three months was analysed using Student's $t$-test for paired measurements. The relationship between the neuroimaging variables and the clinical variables was calculated using a Spearman's rho non-parametric test. Statistical analyses were performed using SPSS for Windows v15, with $p$-values $<0.05$ considered significant. The difference of each metabolite was calculated after treatment with memantine and was 
placed as a dependent variable in a linear regression model after checking the normality of the new variables. Changes in measuring instruments, pain, the HADS, FIQ, MEC and CGI were included as independent variables.

\section{Ethics and consent}

Informed consent was obtained from the participants before they were aware of to which group they were assigned. The study follows the Declaration of Helsinki convention with posterior modifications as well as the Declaration of Madrid of the World Psychiatric Association. The study protocol was approved by the Clinical Research Ethics Committee of Aragón (June 2012).

\section{Results}

The study patient sample showed the expected characteristics of FM patients, including female predominance $(100 \%)$, middle-aged $(M=46.82, S D=5.34)$, married $(8 / 10)$, medium level of education $(M=8.44$ years, $S D=2.16$ years of education) and frequently disabled $(5 / 10)$ or on sick leave $(1 / 10)$.

All the patients completed the study. Six patients took the recommended doses $(20 \mathrm{mg} /$ day), two reached $15 \mathrm{mg} /$ day and the remaining two reached $10 \mathrm{mg} /$ day. Treatment adherence was $>95 \%$ for all patients.
The conventional MR brain parenchyma images were normal in all subjects. The mean values of the metabolite ratios, the absolute value and the correlation coefficient are shown in Table 1, reported by each exploration area. Significant changes between baseline and post-treatment metabolite levels were seen in all the patients. After three months of memantine treatment, the $\mathrm{Glx} / \mathrm{Cr}$ ratio (the main outcome of the study) increased in the anterior insula $(2.03$ vs $2.17 ; r=0.71$; $p=0.022)$ and in the posterior insula (1.77 vs. 2.00; $r=0.82 ; p=0.004)$. There was a significant increase in $\mathrm{Cho} / \mathrm{Cr}$ in the posterior cingulate $(0.18$ at baseline vs. 0.19 after treatment; $r=0.71 ; p=0.023)$; NA+ NAAG increased in the anterior insula (7.56 vs. 7.70 ; $r=0.67 ; p=0.033)$; NAA increased (4.47 at baseline vs. 4.71 after treatment; $r=0.75 ; p=0.02)$ and NAA NAAG also increased (5.89 vs. 5.98; $r=0.82$; $p=0.007)$ in the left hippocampus. In the right hippocampus, $\mathrm{Cr}$ increased (3.60 vs. $3.85 ; r=0.82 ; p=0.007$ ) and NAA+NAAG also increased (5.31 vs. 5.79; $r=0.80 ; p=0.01)$. There was a decrease in the Cho (1.31 vs. $1.29, r=0.83 ; p=0.003)$ and $\mathrm{Cho} / \mathrm{Cr}$ levels in the posterior insula $(0.24$ vs. $0.23 ; r=0.79$; $p=0.006)$, in Cho in the left hippocampus (1.43 vs. $1.42 ; r=0.75 ; p=0.020)$ and in the $\mathrm{Cho} / \mathrm{Cr}$ ratio $(0.34$ vs. $0.33 ; r=0.85 ; p=0.004)$ in the right hippocampus at baseline and again after three months of treatment with memantine.

Table 2 summarises the clinical and psychological outcomes of the study. At the three-month follow-up,

Table 1. Absolute values and creatine ratios of various metabolites in the posterior cingulate, right anterior and posterior insula, and both hippocampi in 10 fibromyalgia patients before treatment and after three months of treatment with memantine.

\begin{tabular}{|c|c|c|c|c|}
\hline & Baseline $(N=10)$ & Post treatment $(N=10)$ & & \\
\hline Region and metabolites & $M(S D)$ & $M(S D)$ & Correlation $(r)$ & $p$-Value* \\
\hline \multicolumn{5}{|l|}{ Posterior cingulate } \\
\hline $\mathrm{Cho} / \mathrm{Cr}$ & $0.18(0.02)$ & $0.19(0.04)$ & 0.71 & 0.023 \\
\hline \multicolumn{5}{|l|}{ Anterior insula } \\
\hline$N A A+N A A G$ & $7.56(0.68)$ & $7.70(0.81)$ & 0.67 & 0.033 \\
\hline $\mathrm{Glx} / \mathrm{Cr}$ & $2.03(0.27)$ & $2.17(0.34)$ & 0.71 & 0.022 \\
\hline \multicolumn{5}{|l|}{ Posterior insula } \\
\hline $\mathrm{Glx} / \mathrm{Cr}$ & $1.77(0.34)$ & $2.00(0.12)$ & 0.82 & 0.004 \\
\hline Cho & $1.31(0.14)$ & $1.29(0.11)$ & 0.83 & 0.003 \\
\hline $\mathrm{Cho} / \mathrm{Cr}$ & $0.24(0.03)$ & $0.23(0.02)$ & 0.79 & 0.006 \\
\hline \multicolumn{5}{|l|}{ Left hippocampus } \\
\hline NAA & $4.47(1.53)$ & $4.71(0.98)$ & 0.75 & 0.020 \\
\hline$N A A+N A A G$ & $5.89(1.12)$ & $5.98(1.29)$ & 0.82 & 0.007 \\
\hline Cho & $1.43(0.18)$ & $1.42(0.25)$ & 0.75 & 0.020 \\
\hline \multicolumn{5}{|l|}{ Right hippocampus } \\
\hline $\mathrm{Cr}$ & $3.60(0.79)$ & $3.85(0.71)$ & 0.82 & 0.007 \\
\hline$N A A+N A A G$ & $5.31(1.12)$ & $5.79(0.96)$ & 0.80 & 0.010 \\
\hline $\mathrm{Cho} / \mathrm{Cr}$ & $0.34(0.04)$ & $0.33(0.02)$ & 0.85 & 0.004 \\
\hline
\end{tabular}

*Non-parametric Wilcoxon's test. Cho/Cr: choline/creatine ratio; NAA: N-acetylaspartate; NAA+NAAG: N-acetylaspartate+Nacetylaspartyl-glutamate; $\mathrm{Gl} / \mathrm{Cr}$ : glutamate+glutamate/creatine ratio. 
Table 2. Psychological outcomes at baseline and at three-month follow-up.

\begin{tabular}{|c|c|c|c|c|c|}
\hline \multirow[b]{2}{*}{ Variable } & \multicolumn{2}{|c|}{ Baseline } & \multicolumn{2}{|c|}{ Three-month follow-up } & \multirow[b]{2}{*}{$p$} \\
\hline & $M$ & $S D$ & $M$ & $S D$ & \\
\hline Pain VAS & 54.00 & 24.92 & 48.00 & 26.99 & 0.504 \\
\hline Pain sphygmomanometer & 86.00 & 30.62 & 104.00 & 50.81 & 0.165 \\
\hline HADS-a & 11.50 & 3.97 & 10.20 & 3.58 & 0.408 \\
\hline HADS-d & 7.70 & 0.81 & 7.56 & 0.68 & $0.042^{\star}$ \\
\hline MEC & 31.50 & 2.95 & 34.40 & 0.69 & $0.005^{\star}$ \\
\hline CGI-Severity & 5.79 & 0.96 & 5.31 & 1.12 & $0.007^{\star}$ \\
\hline FIQ & 58.95 & 17.48 & 49.81 & 20.52 & 0.202 \\
\hline$E Q-5 D$ & 49.50 & 19.50 & 55.50 & 23.85 & 0.330 \\
\hline
\end{tabular}

*Non-parametric Wilcoxon's test: $p<0.05$. HADS: Hospital Anxiety Depression Scale; -a: anxiety; -d: depression; MEC: Cognitive Mini-Exam; CGI-Severity: illness severity; FIQ: Fibromyalgia Impact Questionnaire; EQ-5D: EuroQol 5D questionnaire.

Table 3. Correlation of brain metabolite values and neuropsychological variables in individuals with fibromyalgia after treatment with memantine.

\begin{tabular}{|c|c|c|c|c|c|c|c|}
\hline Variable & Pain & MEC & HADS-a & HADS-d & FIQ & CGI & $R^{2}$ \\
\hline \multicolumn{8}{|c|}{ Posterior cingulate } \\
\hline $\mathrm{Cr}$ & $-0.01(0.001)$ & & $0.06(0.0006)$ & $0.38(0.034)$ & & $-0.14(0.050)$ & $95.89 \%$ \\
\hline $\mathrm{ml}$ & $0.02(0.003)$ & & $0.06(0.014)$ & & $-0.017(0.010)$ & & $88.95 \%$ \\
\hline $\mathrm{ml} / \mathrm{Cr}$ & $0.01(0.0004)$ & $-0.012(0.003)$ & $-0.012(0.0009)$ & $-0.016(0.007)$ & & $0.073(0.012)$ & $97.13 \%$ \\
\hline Cho & $0.06(0.001)$ & & & & $-0.011(0.003)$ & $0.15(0.030)$ & $81.93 \%$ \\
\hline $\mathrm{Glx} / \mathrm{Cr}$ & & $0.11(0.041)$ & & & & & $42.08 \%$ \\
\hline \multicolumn{8}{|c|}{ Anterior insula } \\
\hline $\mathrm{Cr}$ & & & $-0.11(0.025)$ & & & & $72.22 \%$ \\
\hline Cho & & $0.10(0.020)$ & $-0.02(0.007)$ & & & & $77.73 \%$ \\
\hline \multicolumn{8}{|l|}{$\mathrm{Cho} / \mathrm{Cr}$} \\
\hline tNAA & $-0.03(0.010)$ & & & & & & $54.06 \%$ \\
\hline \multicolumn{8}{|c|}{ Posterior insula } \\
\hline $\mathrm{ml} / \mathrm{Cr}$ & & & & $-0.07(0.018)$ & & $0.09(0.026)$ & $67.77 \%$ \\
\hline NAA & & & & $0.078(0.028)$ & $-0.411(0.188)$ & & $87.70 \%$ \\
\hline $\mathrm{NAA} / \mathrm{Cr}$ & $0.010(0.001)$ & $-0.10(0.0006)$ & $0.017(0.0003)$ & $-0.14(0.001)$ & $-0.001(0.0002)$ & $0.15(0.0002)$ & $99.98 \%$ \\
\hline Cho & $0.05(0.001)$ & & $0.013(0.004)$ & $-0.08(0.021)$ & $-0.01(0.002)$ & & $82.77 \%$ \\
\hline $\mathrm{Cho} / \mathrm{Cr}$ & $0.001(0.001)$ & & $0.003(0.001)$ & $-0.02(0.002)$ & $-0.002(0.001)$ & $0.013(0.0004)$ & $93.92 \%$ \\
\hline Glx & & & & & $-0.13(0.031)$ & & $59.92 \%$ \\
\hline $\mathrm{G} \mid x / C r$ & & $0.081(0.028)$ & & & $-0.018(0.004)$ & & $62.68 \%$ \\
\hline \multicolumn{8}{|c|}{ Left hippocampus } \\
\hline $\mathrm{Glu} / \mathrm{Cr}$ & & & $-0.054(0.022)$ & & & & $56.43 \%$ \\
\hline $\mathrm{Cho} / \mathrm{Cr}$ & $-0.001(0.001)$ & & & $0.022(0.0065)$ & & & $58.03 \%$ \\
\hline
\end{tabular}

Cr: creatine; ml: myo-inositol; Cho: choline; tNAA: total N-acetylaspartate+N-acetylaspartate glutamate; Glx: glutamate+glutamine; Glu: glutamate; Glu/ Cr: glutamate/creatine ratio.

FM patients treated with memantine improved in cognitive function. The patients were assessed with the MEC $(M=31.50, S D=2.95$ at baseline vs. $M=34.40$, $S D=0.69$ at three months; $p=0.005)$. Depression was measured with the HADS-d $(M=7.70, S D=0.81$ at baseline vs. $M=7.56, S D=0.68$ at three months; $p=0.042)$, and clinical severity was assessed with the CGI severity scale $(M=5.79, S D=0.96$ at baseline vs. $M=5.31, \quad S D=1.12$ at three months; $p=0.007$ ).
There was no relationship between improvement and the number of pills taken. There was also no relationship between an improvement in psychological variables and changes in any metabolite in any brain region.

Table 3 shows the correlation of brain metabolite values and neuropsychological variables in individuals with FM after treatment with memantine. After analysing metabolites and their correlation with clinical variables, it was found that in the posterior cingulum, 
the increase in $\mathrm{Cr}$ had a significant negative correlation with perceived pain and the CGI (increase in metabolite and decrease in perceived pain and severity of symptoms), Cho augmentation had a negative correlation with the FIQ, and $\mathrm{mI} / \mathrm{Cr}$ augmentation had a significant negative correlation with the MEC, HADS-a and HADS-d. In the anterior insula, the increase in Glu has significant negative correlation with the CGI, the increment of $\mathrm{Cr}$ and Cho had a negative correlation with HADS-a and the increase in total NAA had a negative correlation with perceived pain. In the posterior insula, the increase of $\mathrm{mI} / \mathrm{Cr}$ had a significant negative correlation with HADS-d, the increase in NAA had a significant negative correlation with the MEC and FIQ, the increase in NAA/Cr had a significant negative correlation with the MEC, HADS-d and FIQ, the increment of Cho and $\mathrm{Cho} / \mathrm{Cr}$ had a significant negative correlation with the HADS-d and FIQ and the increase in Glx and Glx/Cr had a significant negative correlation with the FIQ. In the left hippocampus, the increase in $\mathrm{Cho} / \mathrm{Cr}$ had a significant negative correlation with perceived pain.

\section{Discussion}

This study assessed the efficacy of memantine, a NMDA receptor antagonist, in the treatment of FM. The rationale for using memantine was the high levels of Glu found in some brain regions of FM patients. ${ }^{28-30}$ We evaluated the effect of memantine both in brain metabolites and in relevant clinical variables of FM and explored the correlations existing between them.

This ${ }^{1} \mathrm{H}-\mathrm{MRS}$ study has four major findings between baseline and three months post treatment in patients who received memantine: (a) an increase in $\mathrm{Glx} / \mathrm{Cr}$ in the anterior and posterior insula, (b) raised levels of $\mathrm{NAA}$ and NAA+NAAG in both hippocampi and the anterior insula, (c) an increase in $\mathrm{Cr}$ in the right hippocampus and (d) reduced Cho in the posterior insula and both hippocampi.

This study found an increase in the $\mathrm{Glx} / \mathrm{Cr}$ ratio in the anterior and posterior insula three months post treatment (Figure 2).

Previous studies have showed Glu increases in the posterior insula ${ }^{30}$ and left amygdale ${ }^{29}$ in FM patients and increases in Glx/Cr levels in the $\mathrm{PCC}^{28}$ in patients with FM and somatisation disorder.

Although the cause of FM remains inconclusive, there are converging data in favour of a dysregulation of pain processing in the CNS of FM patients, particularly associated with an increase in cerebral Glu levels. Furthermore, there is evidence to support an association between increased Glu levels and an increase in FM symptoms. ${ }^{45}$

A number of authors ${ }^{46,47}$ further support a causal relationship between raised cerebral Glu and FM by demonstrating reversibility. They show that treatment of FM with acupuncture or pregabalin results in a reduction in cerebral Glu levels compared to placebo. Paradoxically, a previous study conducted by our group in patients with FM who were treated with memantine for six months showed an increase in cerebral Glu, Glu/Cr and Glx in the posterior cingulate. However, it has been postulated that this is due to the mechanism of action of the drug, which acts as a receptor antagonist that blocks the action of Glu rather than reducing Glu levels. ${ }^{48}$ The mechanism of action would not be a reduction in the levels of glutamate or its

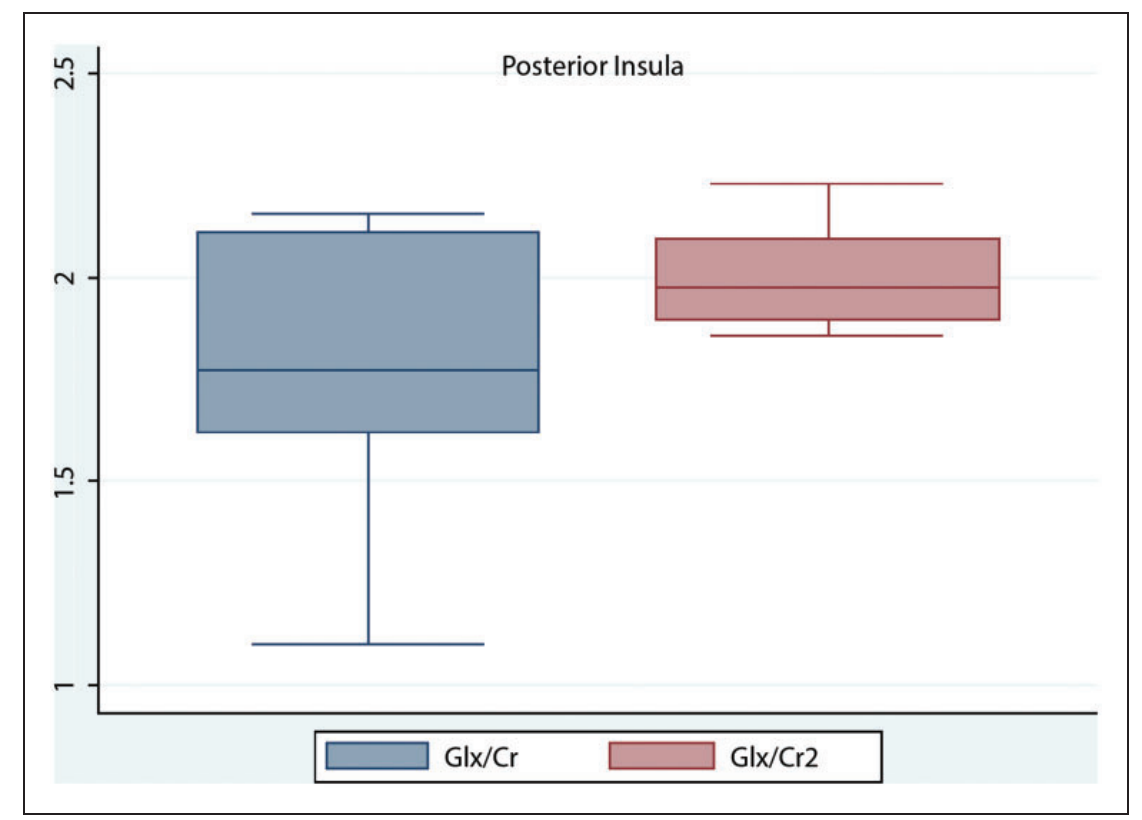

Figure 2. Box plots representing the glutamate+glutamine/creatine ratios $(G \mid x / C r)$ in the posterior insula in fibromyalgia patients who were treated with memantine for three months. $\mathrm{Glx} / \mathrm{Cr}$ represents baseline values, whereas $\mathrm{Glx} / \mathrm{Cr} 2$ represents post-treatment values. 
release. Instead, glutamine would reduce its neurotoxic effect, stopping the entry of excess calcium as it blocks the (NMDA) receptor. ${ }^{49}$

Mechanisms that might increase the combined Glx $(\mathrm{Glu}+\mathrm{Gln}+\mathrm{GABA})$ concentration include greater presynaptic vesicular release of Glu, ${ }^{50}$ faster breakdown of NAAG into NAA and Glu, ${ }^{51}$ slower conversion of Gln to $\mathrm{GABA}^{52}$ and net production rather than consumption of Glu by the Krebs cycle in neurons and astrocytes. $^{53,54}$

It is known that synaptic Glu is taken up by astrocytes, where it is converted to Gln and transported to neurons for the production of Glu or GABA. ${ }^{11,21}$ One theory that has been postulated is that the observed association between Glu levels in the brain and FM is due to a deficiency in astrocyte activity, ${ }^{55}$ although it has recently been postulated that pain in FM may be related to glial activation rather than astrocytes. ${ }^{56} \mathrm{It}$ has generally been described that mean Glx and GABA levels (pooled across regions of interest) correlated positively, indicating that participants with higher levels of Glx also show higher levels of GABA. ${ }^{57}$ The reported ${ }^{1} \mathrm{H}$-MRS levels of Glx therefore reflect the joint neurotransmitter pools, which may be related to both signalling and metabolism.

FM and chronic pain are associated with abnormalities in the levels of certain brain metabolites, including decreases in NAA in both hippocampi, ${ }^{58,59}$ the dorsolateral prefrontal cortex ${ }^{60}$ and the thalamus ${ }^{61}$ and decreases in NAA+NAAG in the hippocampus and the posterior insula. ${ }^{28,62}$ The hippocampus plays a role in memory and cognition, ${ }^{63}$ two functions that may be influenced by prolonged stress. The posterior insula is known to play a prominent role in pain and interoceptive sensory processing, ${ }^{64}$ whereas the anterior insula is involved in the affective processing of pain and other subjective feelings. ${ }^{65}$

We found an increase in NAA and NAA+NAAG levels in both hippocampi and the anterior insula of patients after three months of treatment with memantine compared to the baseline study. NAA is a marker of neuronal density/dysfunction, axonal viability and mitochondrial metabolism. ${ }^{66}$ The function of NAA within axons in the white matter is unknown, but one of its roles may involve the synthesis of neurotransmitters. ${ }^{67}$ There are two reports indicating that postnatal membrane turnover is high with increasing brain concentrations of NAA, $\mathrm{Cr}$ and $\mathrm{PCr}{ }^{68,69}$ The responsiveness of NAA to improved neuron 'health' suggests similar measures of NAA as a potential surrogate for therapeutic efficacy in FM, and increased NAA levels were detected under treatment with memantine (Figure 3). NAAG is co-localised with NAA in neurons and releases NAA and Glu when it is cleaved by $\mathrm{N}$-acetylated alpha-linked dipeptidase. ${ }^{70} \mathrm{Glu}$, and possibly NAAG, are excitatory amino acids. Within physiological concentrations, Glu can be neurotoxic. Data suggest that NAAG may be the form in which the neuron stores Glu in order to protect the cell from the compound's excitatory, and potentially neurotoxic, action. The quantification of NAA by ${ }^{1} \mathrm{H}-\mathrm{MRS}$ could improve understanding of other neurological conditions. For example, lower hippocampal NAA levels suggest a neuronal or an axonal metabolic dysfunction or a combination of these processes. Some studies found that the persistence of elevated $\mathrm{Ca} 2+$ levels in hippocampal neurons exposed to Glu correlated with the extent of neuronal death ${ }^{71}$ and that a large increase

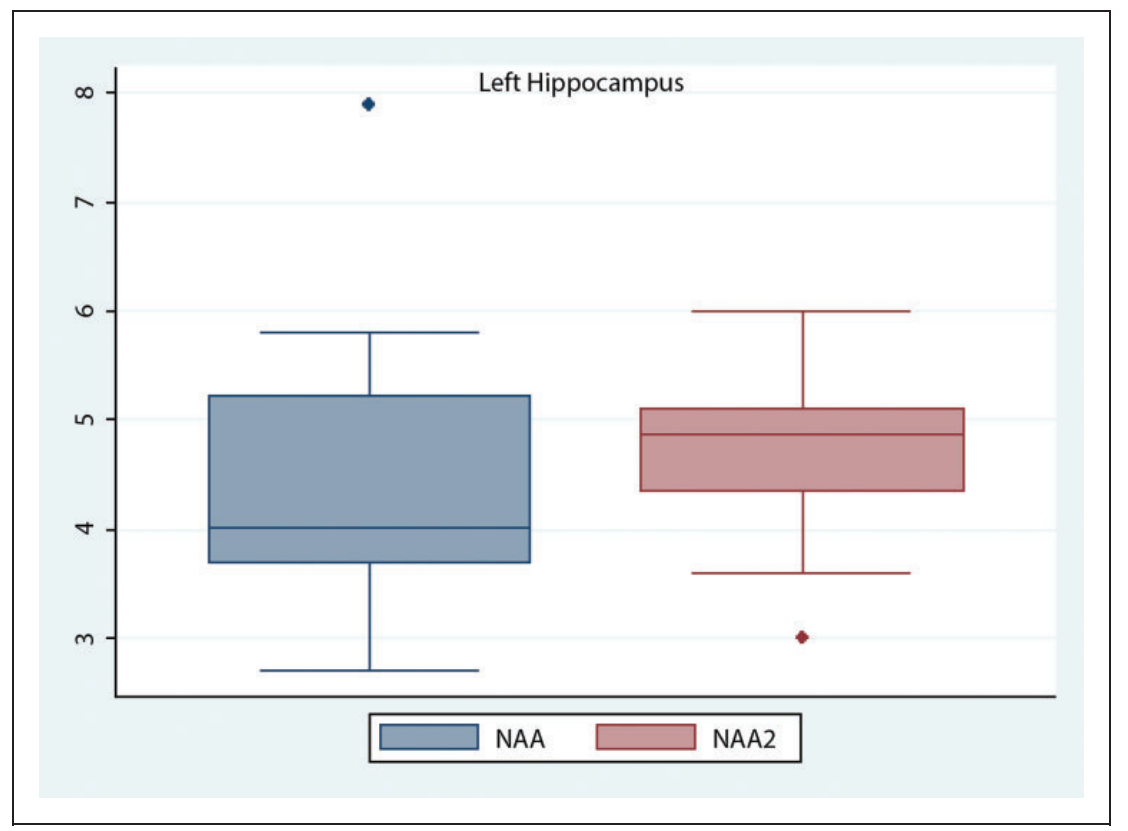

Figure 3. Box plots representing $\mathrm{N}$-acetylaspartate in the left hippocampus in fibromyalgia patients who were treated with memantine for three months. NAA represents baseline values, whereas NAA2 represents the post-treatment values. 
in $\mathrm{Ca} 2+$ in cultured hippocampal neurons after Glu application predicted cell death. ${ }^{72}$ We suggest that hippocampal dysfunction may be partially responsible for some of the phenomena associated with FM. Blocking NMDA receptors in the hippocampal formation reduces nociceptive behaviours. This in turn supports the hypothesis that the hippocampal formation is involved in pain-related neural processing and the expression of pain-related behaviours. ${ }^{73}$ Memantine acts as a neuroprotectant by decreasing Glu excitotoxicity, and it is known to increase levels of brain-derived neurotrophic factor, thus influencing synaptic plasticity in rats and reducing beta-amyloid-induced apoptotic death and neuroinflammation in the hippocampus. ${ }^{74}$

We found a significant elevation of creatine/ phosphocreatine in the right hippocampus, and a statistically insignificant elevation in the posterior, anterior and posterior insula post treatment. Creatine is taken in the diet, and in humans, it is synthesised within the liver, kidneys and pancreas. $\mathrm{Cr}$ and $\mathrm{PCr}$ are measured as a single peak with ${ }^{1} \mathrm{H}-\mathrm{MRS}$. $\mathrm{PCr}$ serve as a reserve for high-energy phosphates in the cytosol of muscle and neurons and buffers cellular adenosine triphosphate/ adenosine diphosphate (ATP/ADP) reservoirs. Tissues such as muscle and brain where the largest changes in energy metabolism occur have the highest concentrations of creatine kinase enzyme. $\mathrm{Cr}$ is used as internal reference value, as it is the most stable brain metabolite. It has a role in the brain's energy supply system and in osmoregulation. $^{36}$

We found a decrease in Cho and Cho/Cr ratios in the posterior insula and both hippocampi post treatment with memantine compared to baseline metabolites. This was caused both by a decrease in Cho and by a significant increase in $\mathrm{Cr}$. Previous studies have shown an increase in $\mathrm{Cho} / \mathrm{Cr}$ in the caudate nucleus and ventrolateral prefrontal cortex of patients with FM compared to healthy controls. ${ }^{28}$ Cho is the precursor for acetylcholine (ACho) and phosphatidylcholine (PtdCho). The synthesis of ACho occurs only within cholinergic neurons, whereas all cells use Cho to synthesise PtdCho, which is a major constituent of the cell membrane. ${ }^{75}$ Cho is a marker of phospholipid metabolism a marker of cellular membrane turnover, reflecting cellular proliferation. Membrane synthesis, however, is an active process, suggesting that Cho levels may also reflect cell energetic status. ${ }^{76}$ Changes in $\mathrm{Cho} / \mathrm{Cr}$ levels (though non-specific) do provide a sensitive indication of altered brain metabolic activity. The exact pathophysiology that could explain altered $\mathrm{Cho} / \mathrm{Cr}$ levels in FM is unclear. ${ }^{28} \mathrm{We}$ also found an increase in $\mathrm{Cho} / \mathrm{Cr}$ in the posterior cingulate of three patients. One hypothesis would be that the second voxel was placed very close to the corpus callosum, which may have altered the values, given that the white matter contains a higher amount of Cho than the grey matter.

In our study, memantine improved several key clinical aspects of FM: cognitive function, depression and overall clinical symptoms. Despite the cognitive dysfunction characteristics of $\mathrm{FM}^{77}$ it is quite different from that of dementia. The effectiveness of memantine in dementia ${ }^{78}$ would lead one also to expect a positive effect on FM. The improvement in depression is more unexpected. Some preliminary reports have described rapid antidepressant effects in treatment-resistant bipolar depression after an intravenous infusion of ketamine, another NMDA receptor antagonist. ${ }^{79}$ However, this is the first time in which a three-month follow-up antidepressant effect has been demonstrated with an NMDA receptor antagonist using oral treatment. In addition to its effect on FM, memantine could possibly be assessed as an add-on treatment for treatment-resistant depression. Previous studies conducted by our research group with patients treated for six months with memantine showed a correlation between the Cho and the FIQ in the posterior insula. ${ }^{66}$ The memantine significantly decreased ratings on a pain VAS and pain measured with a sphygmomanometer. All other secondary outcomes, except anxiety, also improved. ${ }^{80}$

Finally, an improvement in overall clinical symptoms may be foreseen because of the rationale of this study: the raised Glu levels described in FM patients ${ }^{28-30}$ and possibly related to pain and other FM symptoms were supposed to be decreased by an NMDA receptor antagonist. This result has been demonstrated, and we have seen how the clinical improvement of some neuropsychological variables correlates with metabolic changes. Therefore, longer follow-ups are necessary to confirm the time stability of the improvement produced with memantine.

\section{Limitations}

These results should be considered with caution due to some important limitations of this study. This is not a controlled study, and the sample size is small because of its exploratory nature.

\section{Conclusions}

This pilot study has shown that memantine demonstrated spectroscopic effects in patients with FM. Our results demonstrate raised neuronal and axonal metabolic function, or a combination of these processes, supporting the hypothesis that memantine may induce some short-term recovery of neuronal function in brain regions significantly affected by FM. MRS could be useful in monitoring the progress of and response to FM treatment.

\section{Funding}

The study was funded by an unrestricted grant from Ludbeck. The project has received funding from DGA group (B17$17 \mathrm{R})$ and the Network for Prevention and Health Promotion in Primary Care (REDIAPP), grant from the Instituto de Salud Carlos III of the Spanish Ministry of 
Economy and Competitiveness, co-financed with European Union ERDF funds (RD16/0007/0005). The funding source played no part in the study design; the collection; analysis or interpretation of data; the writing of the report; or the decision to submit the paper for publication.

\section{Conflict of interest}

The authors declared no potential conflicts of interest with respect to the research, authorship and/or publication of this article.

\section{ORCID iD}

Javier Garcia Campayo (D) https://orcid.org/0000-0002-37974218

\section{References}

1. Wolfe J, Smythe HA, Yunus MB, et al. American College of Rheumatology 1990 criteria for the classification of fibromyalgia. Report of the Multicenter Criteria Committee. Arthritis Rheum 1990; 33: 160-172.

2. Wolfe F, Clauw DJ, Fitzcharles MA, et al. 2016 Revisions to the 2010/2011 fibromyalgia diagnostic criteria. Semin Arthritis Rheum 2016; 46: 319-329.

3. Branco JC, Bannwarth B, Failde I, et al. Prevalence of fibromyalgia: a survey in five European countries. Semin Arthritis Rheum 2010; 39: 448-453.

4. Garcia Campayo J, Magdalena J, Fernández E, et al. Effectiveness of treatments for fibromyalgia depending of level of care: a meta-analysis. Arthitis Res Ther 2008; 10: R81.

5. Häuser W, Ablin J, Fitzcharles MA, et al. Fibromyalgia. Nat Rev Dis Primers 2015; 1: 15022.

6. Cagnie B, Coppieters I, Denecker S, et al. Central sensitization in fibromyalgia? A systematic review on structural and functional brain MRI. Semin Arthritis Rheum 2014; 44: 68-75.

7. Woolf CJ. Central sensitization: implications for the diagnosis and treatment of pain. Pain 2011; 152: S2-15.

8. Zunhammer M, Schweizer LM, Witte V, et al. Combined glutamate and glutamine levels in pain-processing brain regions are associated with individual pain sensitivity. Pain 2016; 157: 2248-2256.

9. Lee YC, Nassikas NJ and Clauw DJ. The role of the central nervous system in the generation and maintenance of chronic pain in rheumatoid arthritis, osteoarthritis and fibromyalgia. Arthritis Res Ther 2011; 13: 211.

10. Mountz JM, Bradley LA, Modell JG, et al. Fibromyalgia in women. Abnormalities of regional cerebral blood flow in the thalamus are associated with low pain threshold levels. Arthritis Rheum 1995; 38: 926-938.

11. Kwiatek R, Barnden L, Tedman R, et al. Regional cerebral blood flow in fibromyalgia: single-photon-emission computed tomography evidence of reduction in the thalami. Arthritis Rheum 2000; 43: 2823-2833.

12. García-Campayo J, Sanz-Carrillo C, Baringo T, et al. SPECT scan in somatization disorder patients. Aust $N$ Z J Psychiatry 2002; 35: 359-363.

13. Lutz $\mathrm{J}$, Jäger $\mathrm{L}$, de Quervain $\mathrm{D}$, et al. White and gray matter abnormalities in the brain of patients with fibromyalgia: a diffusion-tensor and volumetric imaging study. Arthritis Rheum 2008; 58: 3960-3969.

14. Anselmin M, Catalucci A, Felli V, et al. Diagnostic accuracy of proton magnetic resonance spectroscopy and perfusion-weighted imaging in brain gliomas follow-up: a single institutional experience. Neuroradiol J 2017; 30: 240-252.

15. Splendiani A, Felli V, Di Sibio A, et al. Magnetic resonance imaging and magnetic resonance spectroscopy in a young male patient with anti-N-methyl-D-aspartate receptor encephalitis and uncommon cerebellar involvement: a case report with review of the literature. Neuroradiol $J$ 2016; 29: 30-35.

16. Vigneswaran S, Vera Rojas JH, Garvey L, et al. Differences in the variability of cerebral proton magnetic resonance spectroscopy $\left({ }^{1} \mathrm{H}-\mathrm{MRS}\right)$ measurements within three HIV-infected cohorts. Neuroradiol $J$ 2015; 28: $545-554$.

17. Ranjith G, Parvathy R, Vikas V, et al. Machine learning methods for the classification of gliomas: initial results using features extracted from MR spectroscopy. Neuroradiol J 2015; 28: 106-111.

18. Morales H, Alfaro D, Martinot C, et al. MR spectroscopy of intracranial tuberculomas: a singlet peak at $3.8 \mathrm{ppm}$ as potential marker to differentiate them from malignant tumors. Neuroradiol J 2015; 28: 294-302.

19. Weinbroum AA, Rudick V, Paret G, et al. The role of dextromethorphan in pain control. Can J Anaesth 2000; 47: 585-596.

20. Graven-Nielsen T, Kendall SA, Henriksson KG, et al. Ketamine reduces muscle pain, temporal summation, and referred pain in fibromyalgia patients. Pain 2000; 85: 483-491.

21. Chen HSV and Lipton SA. The chemical biology of clinically tolerated NMDA receptor antagonists. J Neurochem 2006; 97: 1611-1626.

22. Reisberg B, Doody R, Stöffler A, et al. Memantine in moderate-to-severe Alzheimer's disease. $N$ Engl J Med 2003; 348: 1333-1341.

23. Tariot PN, Farlow MR, Grossberg GT, et al. Memantine treatment in patients with moderate to severe Alzheimer disease already receiving donepezil: a randomized controlled trial. JAMA 2004; 291: 317-324.

24. Reisberg B, Doody R, Stoffler A, et al. A 24-week openlabel extension of memantine in moderate to severe Alzheimer's disease. Arch Neurol 2006; 63: 49-54.

25. Nikolajsen L, Gottrup H, Kristensen AGD, et al. Memantine (a N-methyl-D-aspartate receptor antagonist) in the treatment of neuropathic pain after amputation or surgery: a randomized, double-blinded, cross-over study. Anesth Analg 2000; 91: 960-966.

26. Sinis N, Birbaumer N, Gustin S, et al. Memantine treatment of complex regional pain syndrome: a preliminary report of six cases. Clin J Pain 2007; 23: 237-243.

27. Hackworth RJ, Tokarz KA, Fowler IM, et al. Profound pain reduction after induction of memantine treatment in two patients with severe phantom limb pain. Anesth Analg 2008; 107: 1377-1379.

28. Fayed N, Garcia-Campayo J, Magallón R, et al. Localized 1H-NMR spectroscopy in patients with fibromyalgia: a controlled study of changes in cerebral glutamate/glutamine, inositol, choline, and $\mathrm{N}$-acetylaspartate. Arthritis Res Ther 2010; 12: R134.

29. Valdés $M$, Collado A, Bargalló $N$, et al. Increased glutamate/glutamine compounds in the brains of patients with fibromyalgia: a magnetic resonance spectroscopy study. Arthritis Rheum 2010; 62: 1829-1836. 
30. Harris RE, Sundgren PC, Craig AD, et al. Elevated insular glutamate in fibromyalgia is associated with experimental pain. Arthritis Rheum 2009; 60: 3146-3152.

31. Craig AD, Chen K, Bandy D, et al. Thermosensory activation of insular cortex. Nat Neurosci 2000; 3: 184-190.

32. Burgmer M, Pogatzki-Zahn E, Gaubitz M, et al. Fibromyalgia unique temporal brain activation during experimental pain: a controlled fMRI study. J Neural Transm (Vienna) 2010; 117: 123-131.

33. Fayed N, Dávila J, Oliveros A, et al. Utility of different MR modalities in mild cognitive impairment and its use as a predictor of conversion to probable dementia. Acad Radiol 2008; 15: 1089-1098.

34. Modrego P, Fayed N and Sarasa M. Magnetic resonance spectroscopy in the prediction of early conversion from amnestic mild cognitive impairment to dementia: a prospective cohort study. BMJ Open 2011; 1: e000007.

35. Provencher SW. Estimation of metabolite concentrations from localised in vivo proton NMR spectra. Magn Reson Med 1993; 30: 672-679.

36. Danielsen ER and Ross B. Magnetic resonance spectroscopy diagnosis of neurological diseases. New York: Marcel Dekker, 1999, pp.5-22.

37. Vargas A, Vargas A, Hernández-Paz R, et al. Sphygmomanometry-evoked allodynia - a simple bedside test indicative of fibromyalgia: a multicenter developmental study. J Clin Rheumatol 2006; 12: 272-274.

38. Sriwatanakul K, Kelvie W, Lasagna L, et al. Studies with different types of visual analog scales for measurement of pain. Clin Pharmacol Ther 1983; 34: 234-239.

39. Lobo A, Saz P, Marcos G, et al. [Revalidation and standardization of the cognition mini-exam (first Spanish version of the Mini-Mental Status Examination) in the general geriatric population]. Med Clin (Barc) 1999; 112: 767-774.

40. Burckhardt CS, Clark SR and Bennet RM. The Fibromyalgia Impact Questionnaire: development and validation. $J$ Rheumatol 1991; 18: 728-733.

41. Rivera $\mathrm{J}$ and Gonzalez T. The Fibromyalgia Impact Questionnaire: a validated Spanish version to assess the health status in women with fibromyalgia. Clin Exp Rheumatol 2004; 22: 554-560.

42. Tejero A, Guimerá EM, Farré JM, et al. Uso clínico del HAD (Hospital Anxiety and Depression Scale) en población psiquiátrica: un estudio de su sensibilidad, fiabilidad y validez. Rev Dep Psiquiatr Fac Med Barc 1986; 13: 233-238.

43. Badía X, Roset M, Herdman M, et al. La versión española del EuroQol: descripción y aplicaciones. Med Clin (Barc) 1986; 112: 79-86.

44. Guy W, ed. ECDEU assessment manual for psychopharmacology. Rockville, MD: US Department of Health, Education, and Welfare, 1976.

45. Pyke TL, Osmotherly PG and Baines S. Measuring glutamate levels in the brains of fibromyalgia patients and a potential role for glutamate in the pathophysiology of fibromyalgia symptoms. Clin J Pain 2017; 33: 944-954.

46. Harris RE, Sundgren PC, Pang Y, et al. Dynamic levels of glutamate within the insula are associated with improvements in multiple pain domains in fibromyalgia. Arthritis Rheum 2008; 58: 903-907.

47. Harris RE, Napadow V, Huggins JP, et al. Pregabalin rectifies aberrant brain chemistry, connectivity, and functional response in chronic pain patients. Anaesthesiology 2013; 119: 1453-1464.

48. Fayed N, Olivan-Blazquez B, Herrera-Mercadal P, et al. Changes in metabolites after treatment with memantine in fibromyalgia. A double-blind randomised controlled trial with magnetic resonance spectroscopy with a 6-month follow-up. CNS Neurosci Ther 2014; 20: 999-1007.

49. Staud R, Vierck CJ, Robinson ME, et al. Effects of the N-methyl-D-aspartate receptor antagonist dextromethorphan on temporal summation of pain are similar in fibromyalgia patients and normal control subjects. $J$ Pain 2005; 6: 323-332.

50. Williamson LC and Neale JH. Calcium-dependent release of N-acetyl aspartyl glutamate from retinal neurons upon depolarization. Brain Res 1988; 475: 151-155.

51. Cassidy $M$ and Neale JH. N-acetylaspartylglutamate catabolism is achieved by an enzyme on the cell surface of neurons and glia. Neuropeptides 1993; 24: 271-278.

52. Ross B and Blüml S. Magnetic resonance spectroscopy of the human brain. Anat Rec 2001; 265: 54-84.

53. Petroff OAC, Mattson RH and Rothman DL. Proton MRS: GABA and glutamate. Adv Neurol 2000; 83: 261-271.

54. Bejjani A, O'Neill J, Kim J, et al. Elevated glutamatergic compounds in pregenual anterior cingulate in pediatric autism spectrum disorder demonstrated by $1 \mathrm{H}$ MRS and 1H MRSI. PLoS One 2012; 7: e38786.

55. Bak LK, Schousboe A and Waagepeterson HS. The glutamate/ GABA-glutamine cycle: aspects of transport, neurotransmitter homeostasis and ammonia transfer. J Neurochem 2006; 98: 641-653.

56. Albrecht D, Anton Forsberg A, Sandstrom A, et al. Brain glial activation in fibromyalgia. A multi-site positron emission tomography investigation. Brain Behav Immun 2019; 75: 72-83.

57. Zunhammer M, Schweizer L, Witte V, et al. Combined glutamate and glutamine levels in pain-processing brain regions are associated with individual pain sensitivity. Pain 2016; 157: 2248-2256.

58. Wood PB, Ledbetter CR, Glabus MF, et al. Hippocampal metabolite abnormalities in fibromyalgia: correlation with clinical features. J Pain 2009; 10: 47-52.

59. Emad Y, Ragab Y, Zeinhom F, et al. Hippocampus dysfunction may explain symptoms of fibromyalgia syndrome: a study with single-voxel magnetic resonance spectroscopy. J Rheumatol 2008; 35: 1371-1377.

60. Grachev ID, Thomas PS and Ramachandran TS. Decreased levels of $\mathrm{N}$-acetylaspartate in dorsolateral prefrontal cortex in a case of intractable severe sympathetically mediated chronic pain complex regional pain syndrome, type I. Brain Cogn 2002; 49: 102-113.

61. Pattany PM, Yezierski RP, Widerström-Noga EG, et al. Proton magnetic resonance spectroscopy of the thalamus in patients with chronic neuropathic pain after spinal cord injury. Am J Neuroradiol 2002; 23: 901-905.

62. Fayed N, Andres E, Rojas G, et al. Brain dysfunction in fibromyalgia and somatization disorder using proton magnetic resonance spectroscopy: a controlled study. Acta Psychiatr Scand 2012; 126: 115-125.

63. Wood PB. Stress and dopamine: implications for the pathophysiology of chronic widespread pain. Med Hypotheses 2004; 62: 420-424. 
64. Craig AD. How do you feel? Interoception: the sense of the physiological condition of the body. Nat Rev Neurosci 2002; 3: 655-666.

65. Singer T, Seymour B, O'Doherty J, et al. Empathy for pain involves the affective but not sensory components of pain. Science 2004; 303: 1157-1162.

66. Clark JB. N-Acetyl aspartate: a marker for neuronal loss or mitocondrial dysfunction. Dev Neurosci 1998; 20: 271-276

67. Castillo M. Autism and ADHD: common disorders, elusive explanations. Acad Radiol 2005; 12: 533-534.

68. Toft PB, Leth H, Lou HC, et al. Metabolite concentrations in the developing brain estimated with proton MR spectroscopy. J Magn Reson Imaging 1994; 4: 674-680.

69. Kato T, Nishina M, Matshushita K, et al. Neuronal maturation and $\mathrm{N}$-acetyl-L-aspartic acid development in human fetal and child brains. Brain Dev 1997; 19: 131-133.

70. Rothstein JD, Tsai G, Kuncl RW, et al. Abnormal excitatory amino acid metabolism in amyotrophic lateral sclerosis. Ann Neurol 1990; 28: 18-25.

71. Ogura A, Miyamoto M and Kudo Y. Neuronal death in vitro: parallelism between survivability of hippocampal neurones and sustained elevation of cytosolic $\mathrm{Ca} 2+$ after exposure to glutamate receptor agonist. Exp Brain Res 1998; 73: 447-458.

72. Mattson MP, Murrain M, Guthrie PB, et al. Fibroblast growth factor and glutamate: opposing roles in the generation and degeneration of hippocampal neuroarchitecture. J Neurosci 1989; 9: 3728-3740.
73. McKenna JE and Melzack R. Blocking NMDA receptors in the hippocampal dentate gyrus with AP5 produces analgesia in the formalin pain test. Exp Neurol 2001; 172: 92-99.

74. Flores EM, Cappelari SE, Pereira P, et al. Effects of memantine, a non-competitive N-methyl-D-aspartate receptor antagonist, on genomic stability. Basic Clin Pharmacol Toxicol 2011; 109: 413-417.

75. Freeman JJ and Jenden DJ. The source of choline for acetylcholine synthesis in brain. Life Sci 1976; 19: 949-961.

76. Purdon AD, Rosenberger TA, Shetty HU, et al. Energy consumption by phospholipid metabolism in mammalian brain. Neurochem Res 2002; 27: 1641-1647.

77. Glass JM. Review of cognitive dysfunction in fibromyalgia: a convergence on working memory and attentional control impairments. Rheum Dis Clin North Am 2009; 35: 299-311.

78. Raina P, Santaguida P, Ismaila A, et al. Effectiveness of cholinesterase inhibitors and memantine for treating dementia: evidence review for a clinical practice guideline. Ann Intern Med 2008; 148: 379-397.

79. Diazgranados N, Ibrahim L, Brutsche NE, et al. A randomized add-on trial of an N-methyl-D-aspartate antagonist in treatment-resistant bipolar depression. Arch Gen Psychiatry 2010; 67: 793-802.

80. Olivan-Blázquez B, Herrera-Mercadal P, Puebla-Guedea $M$, et al. Efficacy of memantine in the treatment of fibromyalgia: a double-blind randomized controlled trial with 6-month follow-up. Pain 2014; 155: 2517-2525. 